\title{
Proyección sobre energia eléctrica en México mediante la Identidad de Kaya
}

\author{
Projection on electricity in Mexico by Kaya Identity
}

\author{
Edgar R. Sandoval García* "
}

\section{Resumen}

Se analiza al sector energético, con énfasis en la generación de electricidad, por ser una de las fuentes principales que impulsan a la economía, pues se advierte sobre la trascendencia de la energía en el conjunto de la actividad económica del país, donde se muestra que México presenta una fuerte relación entre el crecimiento económico y el uso de energía. Por ello, resulta de suma importancia tener una visión de cuáles serán los requerimientos de electricidad a corto, mediano y largo plazos, para identificar con anterioridad las necesidades y evitar futuras crisis energéticas, como ha pasado en otras partes del mundo, Para llevar a cabo la proyección se utilizó la ecuación llamada "Identidad de Kaya" que nos permite identificar horizontes y respondernos a la pregunta básica de la prospectiva de ¿Qué pasaría si...?

\section{Palabras clave:}

- Producción de electricidad

- Política gubernamental

- Economía de la empresa

\section{Abstract}

We analyze the energy sector, with emphasis on electricity generation, for being one of the main sources that drive the economy, as it warns of the importance of energy in the whole country's economic activity, showing that Mexico has a strong relationship between economic growth and energy use. It is therefore extremely important to have a vision of what the power requirements in the short, medium and long term, to identify needs earlier and avoid future energy crises, as has happened in other parts of the world, to carry out the projection equation was used called "Kaya Identity" horizons allowing us to identify and respond to us to the basic question of foresight of what if ....?

\section{$\mathrm{M}$} éxico es una economía del cual se tiene a nivel mundial grandes expectativas de crecimiento, de hecho, diversos organismos internacionales ${ }^{1}$ pronostican que podría crecer su Producto Interno Bruto (PIB) hasta 4\% anual en las próximas décadas, e inclusive la propuesta presidencial del actual y renovado gobierno es alcanzar una meta de 6 por ciento. $^{2}$

Llevar a cabo tal proeza, requerirá de una fortalecida y bien planeada estructura de soporte, basada en los cambios y ajustes necesarios de diferentes sectores como el político, jurídico, energético, social, entre otros, así como de comportamientos individuales.

\footnotetext{
${ }^{1}$ OCDE (2012), "México, Mejores políticas para un desarrollo incluyente". [En línea]. Disponible http://www.oecd.org/mexico/Mexico\%202012\%20FINALES\%20SEP\%20eBook.pdf

2 Mares, M. (2013), “EPN: crecimiento económico”. [En línea]. Disponible en http://eleconomista.com.mx/columnas/columna-especial-empresas/2013/02/28/epn-crecimiento-economico.
}

* Doctorante en el cinVESTAV del IPN, Transdisciplinario DCTS. esandoval@cinvestav.mx. Una primera versión de " • • este trabajo se presentó en en una sesión del curso "Energía y Desarrollo" del Programa de Maestría del Postgrado de Energía, Facultad de Ingeniería de la unam, el 10 de abril de 2013, bajo la responsabilidad del Prof. Dr. Angel de la Vega Navarro. 
Sin dejar a un lado la importancia de cada uno de los anteriores sectores, analizaremos en este ensayo al energético, y en específico a la generación de electricidad, ya que es una de las fuentes principales que impulsan la maquinaria económica, industrial y de servicios hoy en día, y que se estima su participación se incremente en el futuro, debido a una mayor intervención en el sector transporte.

Así mismo, y de acuerdo con el Balance Nacional de Energía publicado en 2010 por la Secretaría de Energía, México presenta una fuerte relación entre el crecimiento económico y el uso de energía, con un coeficiente de correlación lineal de 0.96 entre el 2000-2010.

\section{Gráfica 1 \\ Correlación uso de energía y desarrollo económico, México 2000-2010}

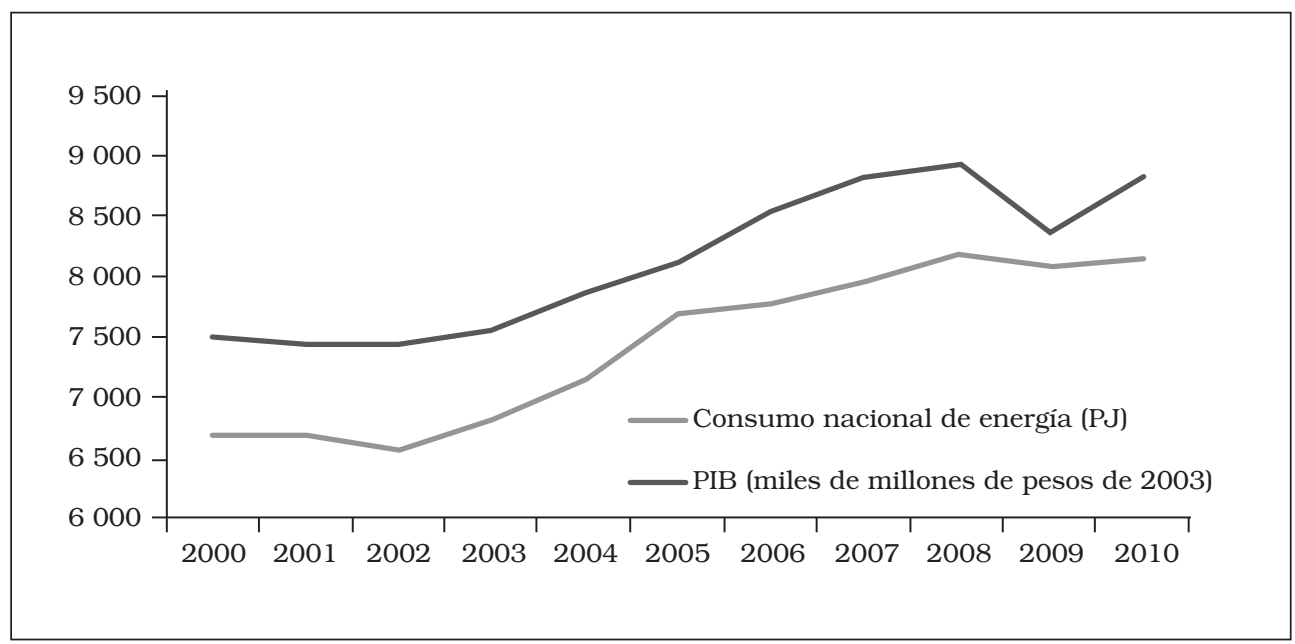

Fuente: Balance Nacional de Energía 2010, sENER.

Tal valor demuestra una marcada sensibilidad del sector energético ante posibles variaciones en el crecimiento económico del país.

Ante tales condiciones y expectativas de crecimiento económico, resultadesumaimportanciatenerunavisióndecuálesseránlos requerimientos de electricidad a corto, mediano y largo plazo, para identificar con anterioridad los posibles cuellos de botella y evitar futuras crisis energéticas como la que puede presentarse por falta de gas natural, o como ha pasado en otras partes del mundo, como el caso de la India ${ }^{3}$ (economía que ha incrementado

${ }^{3}$ Indian power failure shows growing political gridlock, http://www.bloomberg.com/ news/2012-08-02/indian-power-failure-highlights-growing-political-gridlock-view.html. 
8\% al año su PIB PPP entre 2000-2010) en donde 640 millones de personas quedaron sin electricidad por un par de días, en Agosto 2012, con grandes pérdidas económicas.

Para llevar a cabo la proyección de la generación de electricidad necesaria, se utilizó la ecuación llamada "Identidad de Kaya" (Kaya, Y., 1990), la cual ha sido utilizada para el planteamiento de diferentes escenarios referentes a la reducción de emisiones de carbón por parte del Panel Intergubernamental sobre Cambio Climático (IPCC, por sus siglas en inglés). Dicha ecuación plantea que el impacto ambiental por emisiones de carbón, es producto de la variación poblacional, PIB per cápita, intensidad energética (cantidad de energía requerida para producir un peso de PIB) e intensidad de carbono.

Emisiones $\mathrm{CO}_{2}=$ Población $* \mathrm{PIB} /$ Población * Energía $/ \mathrm{PIB} * \mathrm{CO}_{2} /$ Energía

O en una versión corta: $\mathrm{CO}_{2}=P * g * e * c$

Entonces si lo que deseamos es llegar a ser una Economía Verde, bajo un proyecto de crecimiento económico sustentable y socialmente incluyente, y por lo tanto reducir las emisiones de carbono y eliminar el alto índice de pobreza, no es posible reducir la variable $g$ (PIB per cápita). Al contrario, deberá crecer a lo largo del tiempo. Tampoco se puede restringir el incremento poblacional y mucho menos hoy en día cuando la tasa de población activa es una de nuestras "ventajas competitivas". La forma en que podemos crecer económicamente pero con bajas emisiones, es mediante una mejora significativa en la eficiencia productiva y energética, así como en el uso de tecnologías con mínimo nivel de emisiones.

Dado que el estudio propuesto se ha realizado sólo para el sector eléctrico, se adaptó la ecuación (1) de la siguiente manera:

$$
\begin{gathered}
\text { Emisiones eléctricas } \mathrm{CO}_{2}=\text { Población } * \text { PIB } / \text { Población } * \\
\text { Producción eléctrica/PIB } * \mathrm{CO}_{2 \text { eléctricos }} / \text { Producción eléctrica }
\end{gathered}
$$

A continuación se describen cada una de las variables de la ecuación (3) y los supuestos que sustentan su variación en el tiempo. 


\section{Población}

Acorde a datos basados en tendencias de mortalidad y fertilidad obtenidos por el Consejo Nacional de Población (Conapo), México tendrá una tendencia de crecimiento hasta la década entre 2040-2050, para posteriormente empezar a decrecer.

Tales datos se corroboran con las tendencias presentadas por la Organización de las Naciones Unidas, que predicen al 2100 en una de sus variantes (Low-Variant) un comportamiento similar al obtenido por el Conapo. Así mismo en dicho estudio, la onu plantea otros escenarios, obteniendo curvas de comportamiento poblacional, denominadas Medium-Variant, HighVariant y Constant-fertility-Variant (ver gráfica 2).

Para nuestro estudio se utilizaron los datos de la curva Medium-Variant, por recomendación propia del organismo, que indican que la población en México al año 2050 y 2100 será de 143925000 personas y 127081000 personas respectivamente.

\section{Gráfica 2}

\section{Crecimiento de la población en México, 2010-2100}

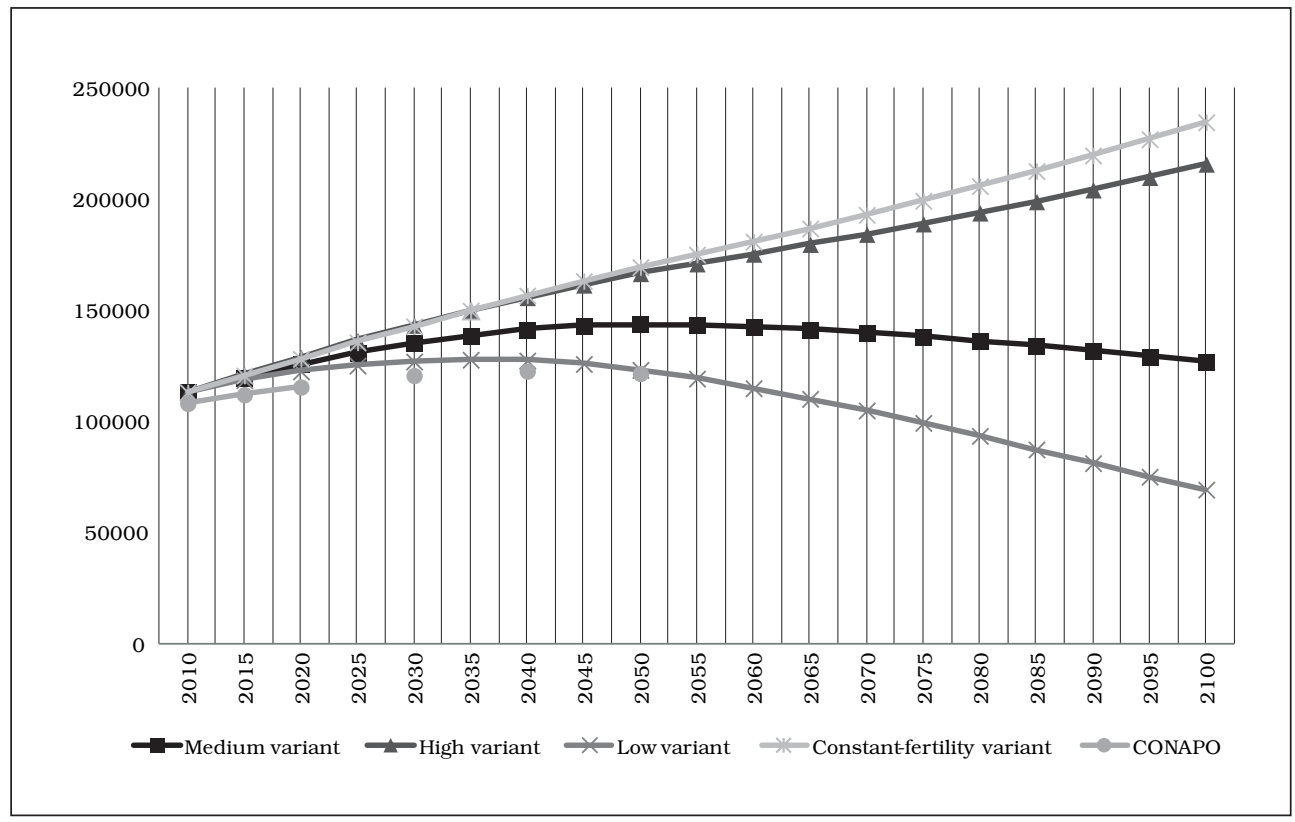

Fuente: elaboración propia con datos de onv y Conapo. 


\section{PIB per cápita}

Si bien esta variable no nos da una versión de la realidad del país en donde vivimos, dada la tremenda desigualdad que rige nuestra sociedad, es un indicador a nivel global que nos permite un nivel de comparación con otras economías en términos de pobreza, calidad de vida, capacidad de compra, entre otros. A lo largo de su historia moderna, la economía mexicana ha mantenido un crecimiento constante, aunque no en el nivel que la sociedad lo ha requerido (gráfica 3).

\section{Gráfica 3}

\section{Comparativo Chile-México-Rep. Corea, PIB per cápita, PPP US\$ de 1990}

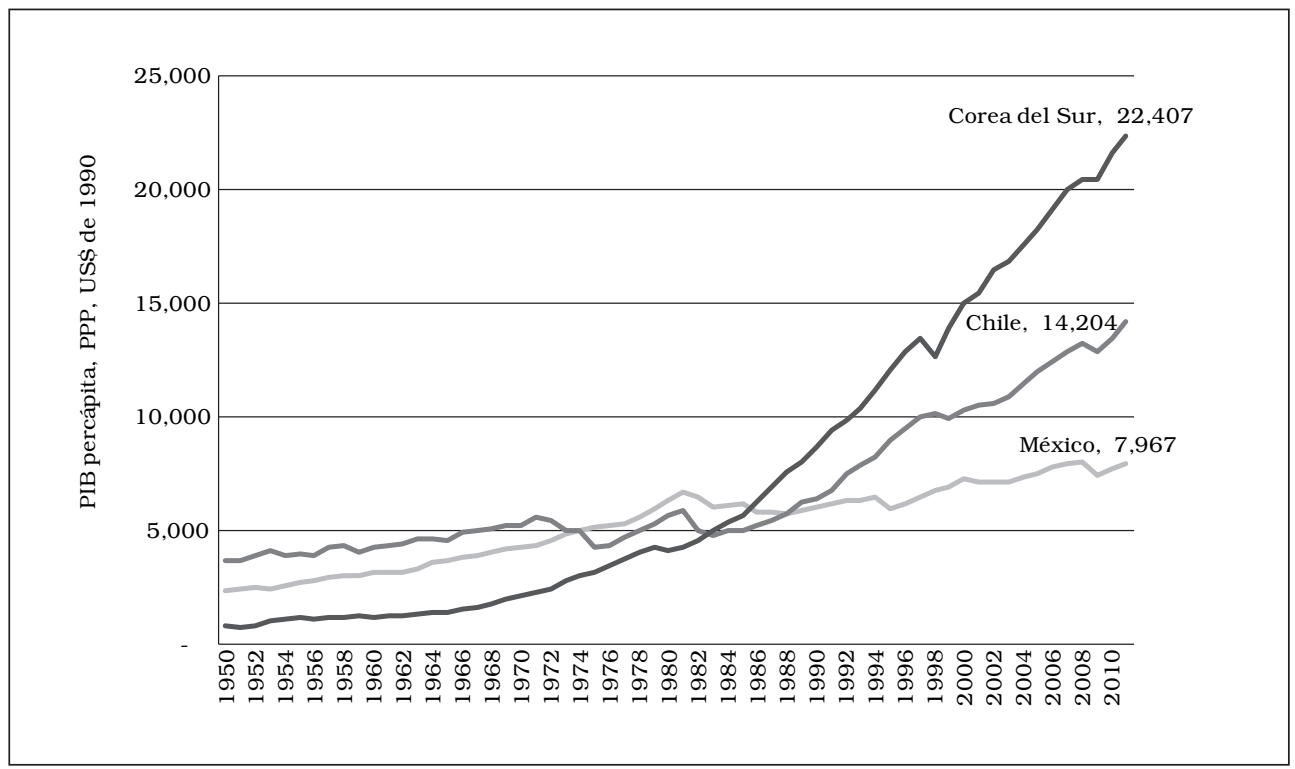

Fuente: elaboración propia con datos de Total Economy Database (TEDb).

En el presente estudio se han propuesto tres escenarios de crecimiento económico, con tasa anual del 2, 4 y $6 \%$ proyectadas al año 2100 , tomando como punto de inicio el PIB de 2011 de \$1 074,127.1 millones de dólares (current US\$, World Bank) ${ }^{4}$ y un PIB per cápita de \$9 442.12 dólares.

\footnotetext{
${ }^{4}$ La proyección al año 2050 y 2100 se hacen en base a los \$1 074127.1 millones de dólares corrientes, con lo que eso implica.
} 
Los valores meta así obtenidos se muestran a continuación en la Cuadro 1.

\section{Cuadro 1 \\ PIB estimado bajo tres escenarios de crecimiento (millones de US\$)}

\begin{tabular}{|r|r|r|}
\hline Año objetivo & 2050 & 2100 \\
\hline Crecimiento PIB 2\% & $\$ 2279618.57$ & $\$ 6135794.04$ \\
\hline Crecimiento PIB 4\% & $\$ 4767849.65$ & $\$ 33883597.70$ \\
\hline Crecimiento PIB 6\% & $\$ 9832830.18$ & $\$ 181122248.83$ \\
\hline
\end{tabular}

Así mismo los valores proyectados de PIB per cápita bajo los anteriores escenarios de crecimiento económico y la población calculada por la onU-Medium Variant son:

\section{Cuadro 2 \\ PIB per cápita proyectado (dólares)}

\begin{tabular}{|r|c|c|}
\hline Año objetivo & 2050 & 2100 \\
\hline PIB per cápita (E-2\%) & $\$ 15838.9$ & $\$ 48282.5$ \\
\hline PIB per cápita (E- 4\%) & $\$ 33127.2$ & $\$ 266629.9$ \\
\hline PIB per cápita (E- 6\%) & $\$ 68319.1$ & $\$ 1425250.4$ \\
\hline
\end{tabular}

\section{Intensidad de carbón ( $\mathrm{CO}_{2 e l e ́ c t r i c o s} /$ Demanda eléctrica)}

Acorde a datos del Banco Mundial y sener, las emisiones totales en México de $\mathrm{CO}_{2}$ alcanzaron las 407300 kTon en 2011 de las cuales, 29.2\% del total fueron generadas por el Sector Eléctrico Nacional (sEN). Cabe aclarar que durante la primera década del siglo xxi el SEN participó en promedio con el $28 \%$ de las emisiones totales de $\mathrm{CO}_{2}$.

Por otro lado, a finales del 2012 entro en vigor la Ley General de Cambio Climático, la cual plantea que al año 2050 se tendrán que abatir en un 50\% las emisiones de carbono respecto a niveles del año 2000. Por lo tanto, tomando en 


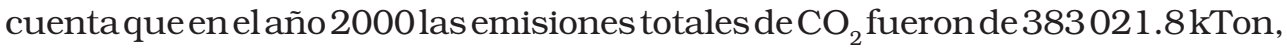
entonces tenemos el compromiso al 2050 de abatir las emisiones a niveles de $53623.1 \mathrm{kTon}$ de $\mathrm{CO}_{2}$ procedentes sólo de la generación de energía eléctrica.

Cabe aclarar que en el presente estudio se considera que del año 2050 al 2100 las emisiones de $\mathrm{CO}_{2}$ permanecen constantes, además que la intensidad de carbón calculada a 2011 fue de (118 931.6 kTon $\mathrm{CO}_{2} / 258,128$ $\mathrm{GWh})=0.4607 \mathrm{kTon} / \mathrm{GWh}$.

\section{Demanda eléctrica/PIB (intensidad eléctrica)}

La demanda eléctrica nacional ha crecido a una tasa acumulada de 5.8\% anual desde 1971 al 2011, de acuerdo a datos del Banco Mundial. En este nuevo siglo la tasa de crecimiento fue de 3.57\% en el período 2001-2011. En cuanto al comportamiento de lo que en este estudio se considera como Intensidad Eléctrica (Producción eléctrica/PIB) ha mantenido un comportamiento a la baja desde finales del siglo xx y principios del siglo xxI, tal como se muestra en el gráfico 4.

\section{Gráfica 4 Intensidad Eléctrica}

(kWh-año/ PIB, miles de dólares corrientes)

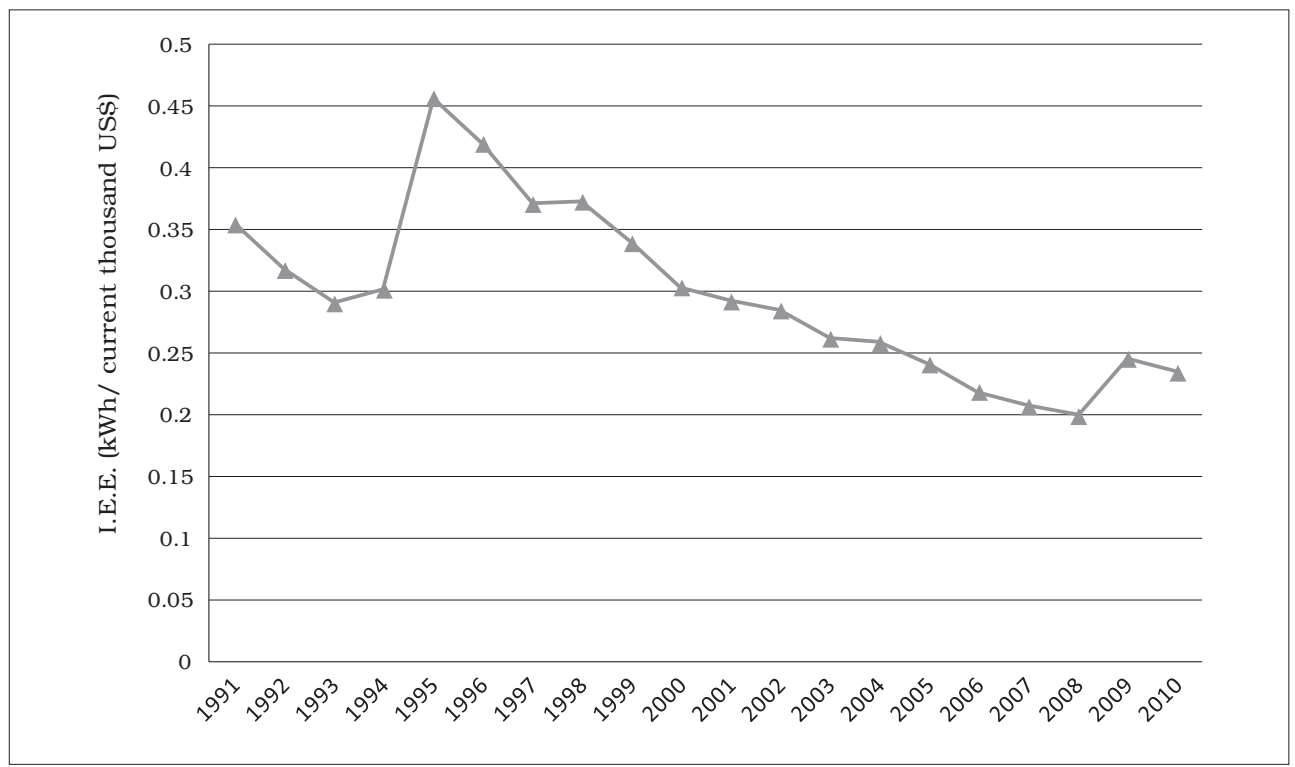

Fuente: elaboración propia con datos del wB, TEDb, IMF. 
Durante el desarrollo del estudio se evaluaron tres comportamientos de intensidad eléctrica (IE) en cada uno de los escenarios de crecimiento económico. El primero es mantener constante la IE a valores del 2011 al 2050 y 2100 (0.24 GWh/millones de dólares corrientes).
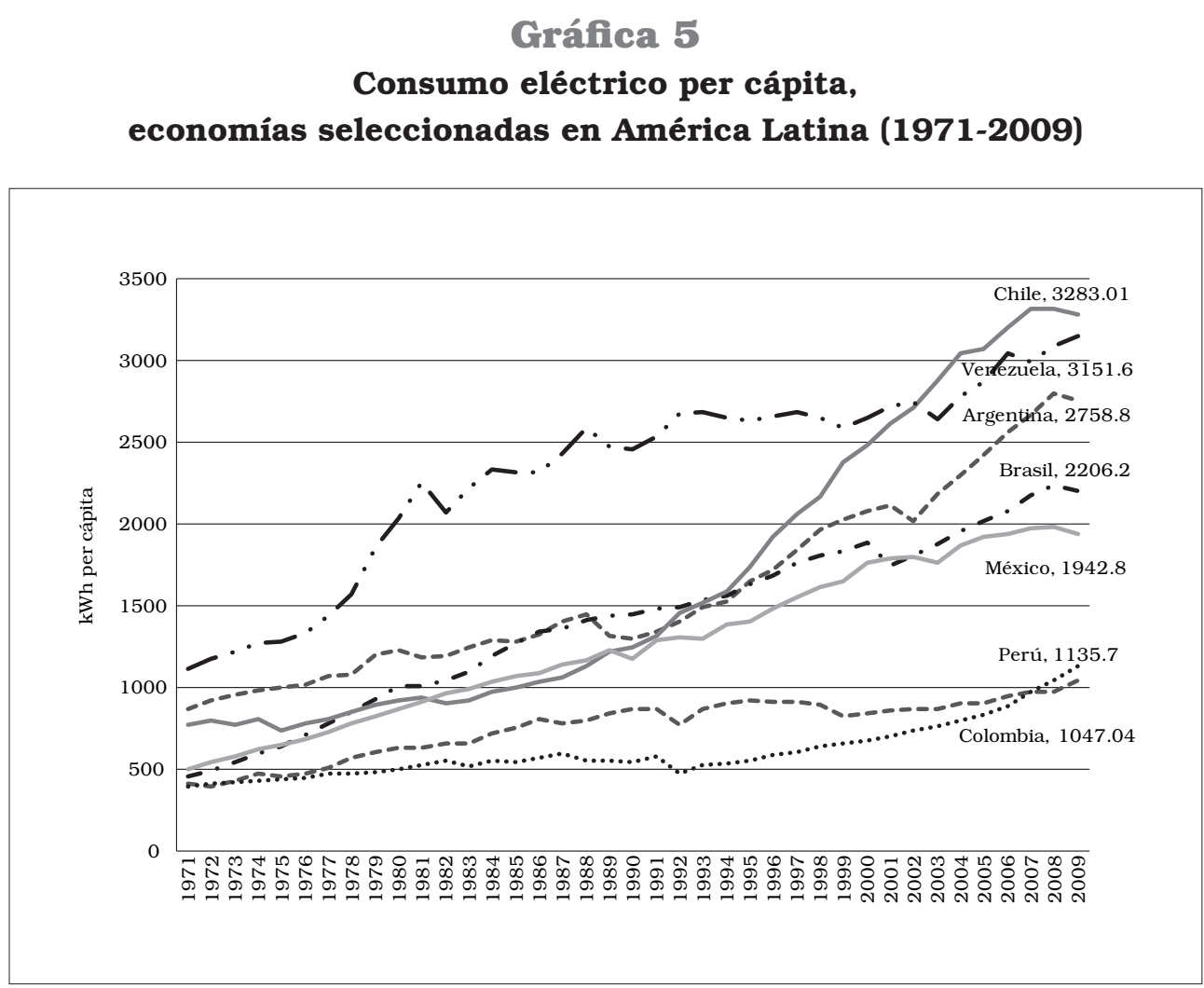

Fuente: elaboración propia con datos del Banco Mundial.

El segundo comportamiento, fue el de incrementar 25\% la IE -como el diferencial en el valor absoluto de 2010 entre Suiza y México- al 2050 y mantenerlo constante hasta el 2100 (0.3 GWh/millones de dólares corrientes). Hay que tener en cuenta que dicho planteamiento se puede prestar a diversos supuestos, por ejemplo el de aumentar los kWh per cápita y reducir el PIB per cápita, o el de incrementar sólo el consumo per cápita y dejar constante el PIB per cápita. Las condiciones hacia donde deberíamos evolucionar es que exista un incremento en el uso per cápita de energía eléctrica -lo que significaría progresar hacia mejores condiciones de vida en términos de educación, salud y equidad-, además de incrementar el ingreso per cápita. 


\section{Gráfica 6 \\ Comparación kWh per cápita en 2010 , diversas economias.}

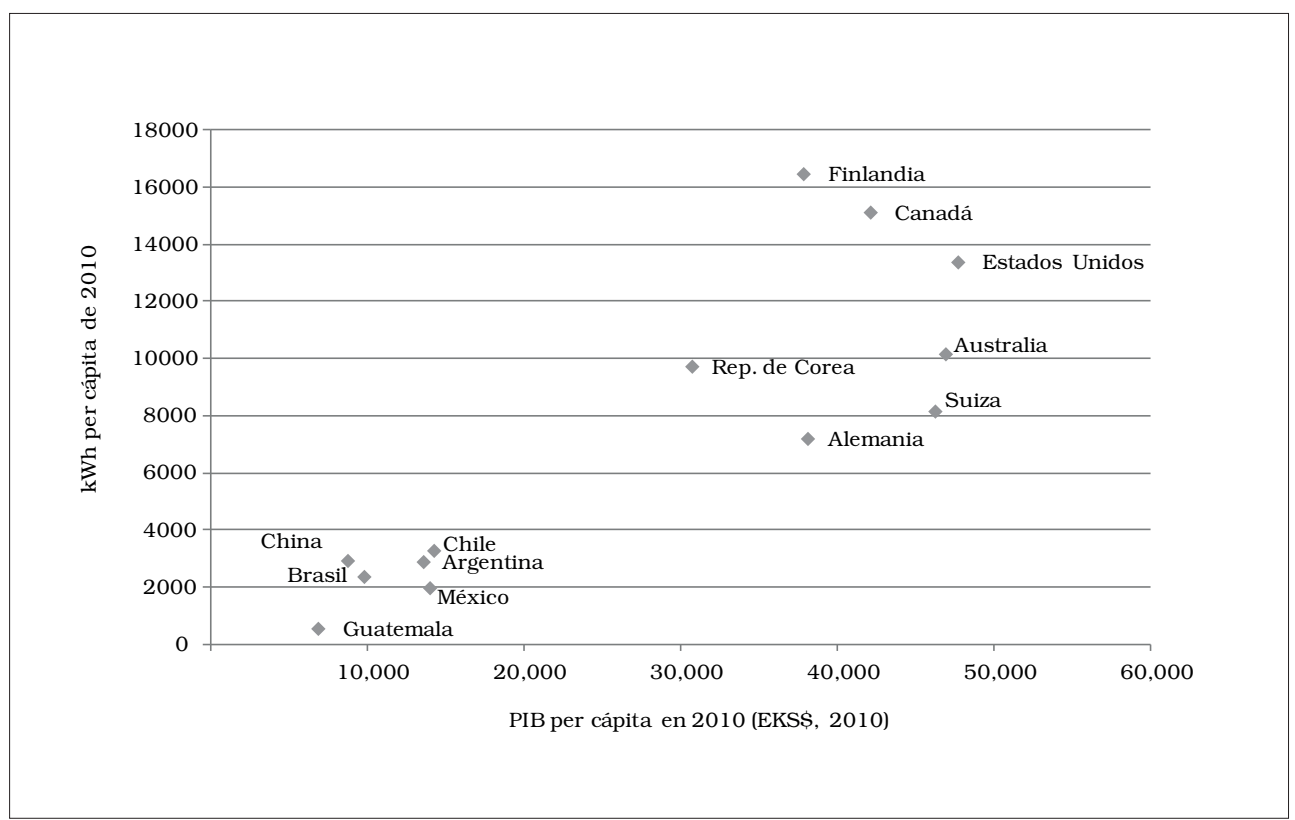

Fuente: elaboración propia con datos del Banco Mundial y TEDb.

El tercer y último comportamiento fue el de reducir 25\% la IE, considerando la reducción en términos de eficiencia energética y productividad, y no de un incremento en términos de pobreza energética, tal como en la actualidad presenta el país. El valor objetivo al 2050 es de 0.18 GWh-año/millones de dólares corrientes, y se mantiene constante al 2100.

\section{Procedimiento}

Mediante una hoja de cálculo se estimó, con un rango de error del +/- 0.01\%, cuál sería el valor de producción eléctrica a futuro que permitiría cumplir con la Identidad de Kaya bajo las consideraciones antes planteadas. 


\section{Cuadro 3 \\ Evaluación de producción eléctrica requerida en hoja de cálculo}

\begin{tabular}{|c|c|c|c|c|c|c|}
\hline \multicolumn{2}{|c|}{ Crecimiento PIB $2.0 \%$ al 2005} & $\$ 2279618.57$ & millones usd & Cálculado & & \\
\hline \multicolumn{2}{|c|}{ PIB real, 2011} & $\$ 1074127.1$ & millones usd & & & \\
\hline \multicolumn{2}{|c|}{ PIB per cápita México } & $\$ 14370.7$ & usd, 2011 & & & \\
\hline \multicolumn{2}{|c|}{$\begin{array}{l}\text { Crecimiento PIB } 2.0 \% \\
\text { al } 2010\end{array}$} & $\$ 6135794.04$ & millones usd & & & \\
\hline \multicolumn{2}{|c|}{ Kton CO2 totales (2000) } & 383021.8 & & & & \\
\hline \multicolumn{2}{|c|}{ Kton CO2 eléctricos (2000) } & 107246.1 & & & & \\
\hline \multicolumn{2}{|c|}{ Kton CO2 eléctricos (2050) } & 53623.1 & & & & \\
\hline \multicolumn{2}{|c|}{ Kton $\mathrm{CO} 2$ eléctricos $(2100)$} & 53623.1 & & & & \\
\hline \multicolumn{2}{|c|}{ GWh/año (2050) } & 547100.0 & \multicolumn{4}{|c|}{ Supuesta para que se cumpla la identidad al 2050} \\
\hline \multicolumn{2}{|c|}{ GWh/año (2100) } & 1472500.0 & \multicolumn{4}{|c|}{ Supuesta para que se cumpla la identidad al 2100} \\
\hline & Población & $\begin{array}{l}\text { PIB per cápita, } \\
2050\end{array}$ & (GW/año)/PIB & $\begin{array}{l}\text { Kton } \mathrm{CO} 2 / \\
\text { (GW/año) }\end{array}$ & & \\
\hline \multirow[t]{2}{*}{$\mathrm{F}=$} & 143925000.0 & 0.01584 & 0.240 & 0.09801326 & $=$ & 53623.9 \\
\hline & Población & $\begin{array}{l}\text { PIB per cápita, } \\
2100\end{array}$ & (GW/año)/PIB & $\begin{array}{l}\text { Kton } \mathrm{CO} 2 / \\
\text { (GW/año) }\end{array}$ & & \\
\hline $\mathrm{F}=$ & 127081000.0 & 0.04828 & 0.240 & 0.036416367 & $=$ & 53626.4 \\
\hline
\end{tabular}

Los resultados así obtenidos se presentan a continuación

\section{Resultados}

El planteamiento de la Identidad de Kaya para el año inicial de 2011 es:

\section{Datos:}

Población: 113 735,000 personas

PIB: \$1 074127.1 millones de dólares, corrientes.

Demanda eléctrica: 258128 GWh-año 
Para el año 2050, la demanda eléctrica (GWh-año) requerida sería de:

\begin{tabular}{|l|c|c|c|}
\hline $\begin{array}{l}\text { IE (GWh-año/millones } \\
\text { de dólares corrientes) }\end{array}$ & 0.18 & 0.24 & 0.3 \\
\hline Crecimiento 2\% PIB & 410330.0 & 547100.0 & 683900.0 \\
\hline Crecimiento 4\% PIB & 858200.0 & 1144300.0 & 1430400.0 \\
\hline Crecimiento 6\% PIB & 1770000.0 & 2360000.0 & 2950000.0 \\
\hline
\end{tabular}

Para el año 2100, la demanda eléctrica (GWh-año) requerida sería de:

\begin{tabular}{|l|c|c|c|}
\hline $\begin{array}{l}\text { IE (GWh-año/millones } \\
\text { de dólares corrientes) }\end{array}$ & 0.18 & 0.24 & 0.3 \\
\hline Crecimiento 2\% PIB & 1104400.0 & 1472500.0 & 1840600.0 \\
\hline Crecimiento 4\% PIB & 6099000.0 & 8132000.0 & 10165000.0 \\
\hline Crecimiento 6\% PIB & 32600000.0 & 43470000.0 & 54339000.0 \\
\hline
\end{tabular}

\section{Discusión}

Considerando el escenario deseable de crecimiento económico a una tasa anual de $6 \%$ y un incremento en la intensidad eléctrica a $0.3 \mathrm{GWh}$-año/millones US\$ (necesaria y fundamental para soportar tal nivel de crecimiento), la generación de electricidad tendría que crecer en 11.5 veces al año 2050 y 210.5 veces al 2100 , respecto de 2011 .

Bajo un escenario moderado de crecimiento económico de $4 \%$ anual y manteniendo una intensidad energética constante desde el 2011, se requeriría un crecimiento en la generación eléctrica de 4.4 veces al año 2050 y 31.5 veces al año 2100, respecto del 2011.

Tomando en cuenta el crecimiento real de las últimas décadas del PIB nacional, en donde han existido años de crecimiento de hasta 6.7\% (1997), pero también ha habido decrecimientos de hasta $-6.12 \%$ (2009), el escenario más plausible sería el de un crecimiento económico de $2 \%$ anual y un crecimiento obligado en la intensidad eléctrica de al menos 25\% respecto 2011. 
La generación eléctrica tendría que incrementarse 2.65 veces al 2050 y 7.1 veces al 2100, respecto del 2011.

\section{Conclusiones}

La "Identidad de Kaya" adoptada por el IPCC, para la definición de escenarios, nos permite identificar horizontes y respondernos a la pregunta básica de la prospectiva ¿Qué pasaría si....? Si bien no es una garantía el que lleguemos a los valores obtenidos, sí nos da una idea de las cantidades necesarias para alcanzar las metas propuestas. Lo importante es identificar con la ayuda de expertos (sociales y tecnólogos) cuál sería la mejor ruta y cómo llegar a buen término. Es decir qué cambios culturales y tecnológicos requeriría el país para enfrentar las posibles adaptaciones que tendríamos que hacer en la vida diaria, en la forma de usar, consumir y ahorrar la energía eléctrica, tomando en cuenta los compromisos internacionales que ha pactado el país respecto a reducción de emisiones.

Si bien, el grueso de la población cuenta con acceso a la energía eléctrica ( $>97 \%$, de acuerdo a la Comisión Federal de Electricidad), somos una sociedad con gran pobreza energética, por lo que, cualquier intento de crecimiento económico, deberá ir de la mano con un incremento per cápita de consumo eléctrico, ya que esto, tal como sucede en economías desarrolladas, significa mejoras en la calidad de vida de la población en general.

Es necesario determinar en un siguiente paso, bajo los escenarios planteados, qué tipo de combustible y de tecnología (convencional, renovable o en conjunto) nos permitirá cumplir con la demanda eléctrica que necesitará el crecimiento económico del país, tomando en cuenta el avance tecnológico, el riesgo financiero por la variación de precios de los combustibles en el futuro y el cumplimiento de los lineamientos globales sobre cambio climático. Será importante además definir las tecnologías que permitieran cumplir nuestros objetivos, y al mismo tiempo ser desarrolladas de manera local para así potenciar una nueva industria nacional.

Así mismo es fundamental que el ingreso per cápita no crezca sólo por el hecho de que la población disminuya en el futuro, si no porque en general, existan mejoras en políticas públicas que promuevan mayores ingresos y poder de compra a la sociedad en general. 


\section{Bibliografía}

conapo (2006), "Proyecciones la población de México 2005-2050". [En línea], ddisponible en http://www.conapo.gob.mx/work/models/CONAPO/proyecciones_ estatales/Proy05-50.pdf

IPCC Special Report, “Emissions Scenarios”. [En línea], disponible en http://www. ipcc.ch/ipccreports/sres/emission/index.php?idp=50

Kaya, Y. (1990), Impact of Carbon Dioxide Emission Control on GNP Growth: Interpretation of Proposed Scenarios, Paper presented to the IPCC Energy and Industry Subgroup, Response Strategies Working Group, Paris.

Population Division of the Department of Economic and Social Affairs of the United Nations Secretariat, World Population Prospects: The 2010 Revision, http://esa. un.org/unpd/wpp/index.htm

Secretaría de Energía (2010), "Balance Nacional de Energía 2010”. [En línea], disponible en http://www.sener.gob.mx/res/PE_y_DT/pub/2011/Balance\%20Nacional\%20de\%20Energ\%C3\%ADa\%202010_2.pdf

The Conference Board, "Total Economy Database”. [En línea], disponible en http:// www.conference-board.org/data/economydatabase/ 